\title{
SDU
}

\section{Externalities, incentives, government failure, and the Coronavirus outbreak}

\author{
by
}

\section{Thiago de Oliveira Souza}

Discussion Papers on Business and Economics

No. $12 / 2020$

FURTHER INFORMATION Department of Business and Economics Faculty of Business and Social Sciences University of Southern Denmark Campusvej 55, DK-5230 Odense M Denmark 


\title{
Externalities, incentives, government failure, and the Coronavirus outbreak
}

\author{
Thiago de Oliveira Souza \\ University of Southern Denmark
}

November 2, 2020

\begin{abstract}
This paper derives and simulates a compartmental model of the Coronavirus outbreak in which individuals have self-interested reactions to the threat of infection, proportional to the heterogeneous risk of complications that they face. As long as high-risk individuals perceive infection as sufficiently undesirable, the externalities created by the free circulation of low-risk individuals are positive and potentially reduce the total number of infections by approximately 100 million in the U.S. (including every high-risk individual). In this case, the social interaction of low-risk individuals should be subsidized, according to the same market failure arguments used to justify broad confinement mandates, which constitute government failures.
\end{abstract}

JEL Codes: D62, H4, C02, I10

Keywords: Externalities, government failure, Coronavirus, Covid-19, pandemic.

Department of Business and Economics, University of Southern Denmark, Campusvej 55, 5230 Odense M, Denmark. Email: tsouza@sam.sdu.dk. 


\section{Introduction}

When externalities exist, free enterprise produces more of the negative ones and less of the positive ones than is socially optimal. This fact becomes a valuable tool to expand government power, with a potential to justify any policy. Nevertheless, we know since at least McKean (1965) and Wolf (1979) that the private incentives of the individuals that constitute "the government" can also diverge substantially from the social optimal. In this case, intervention leads to government failures, as the evidence in Dawson and Seater (2013) and de Oliveira Souza (2020) suggests, for example.

Based on this theoretical framework, the present paper investigates whether the government actions during the Coronavirus outbreak in the U.S. can be justified on the grounds of market failures. In particular, I analyze whether broad government-imposed confinement policies can be fully justified based on the negative externalities that the circulation of self-interested low-risk individuals supposedly have on self-interested high-risk individuals. The short answer is no.

The analysis is based on simulations of an epidemic compartmental model, similar to the original susceptible-infected-recovered model of Kermack and McKendrick (1927),

but including compartments for the number of individuals in hospitals, critical care, and deceased. I also allow government policies to change the rate at which new infections occur, similar to the combination of mitigation and suppression strategies in Ferguson et al. (2020). Both types of models yield similar general results (Eubank et al., 2020), despite the more detailed approach of Ferguson et al. (2020).

In terms of modeling the epidemic, the main innovation of the present paper is to endogenize the rate of infection complications in the population, for which age is a proxy: There are heterogeneous responses to infection risk due to heterogeneous expected loses from infection. I calibrate individual responses to the risk of infection based on government choices observed during the crisis, which I assume to reflect the preferences of the median individual. 
As Eubank et al. (2020) explains, the basic reproductive number, $r_{0}$, is not only a biologically determined characteristic: Transmissibility depends on a combination of biological, environmental, behavioral, and social characteristics, yet most of these variables are largely missing from compartmental models. Indeed, already in Kermack and McKendrick (1927) the constant reproductive number is just a special case within a more general time-varying setting, but still completely exogenous. While there are examples of models that endogenize $r_{0}$ based on government choices (Ferguson et al., 2020; Halloran et al., 2008; Ferguson et al., 2006), I expand this discussion to endogenize the number of complications in the population that arise from infection based on the free choices of self-interested individuals.

The most important modeling assumption that I make is that the individuals that are most at risk have the strongest incentive to adjust their behavior and the strongest reaction to the threat of infection. Based on the hypothesis that government choices reveal the preferences of the median individual (which is below 39 years old in the U.S.), I assume that individuals above 49 years old would, at minimum, choose to self-isolate for the duration of the pandemic to avoid the much higher risk of infection complications that they face. The remaining individuals do not change their behavior. Based on these assumptions, the age distribution in the U.S., and the epidemiological inputs from Ferguson et al. (2020), I show that the pandemic would end before July in the absence of government intervention, with around 60 thousand deaths. There is a peak of around 35 thousand critical cases, compared to little over 45 thousand available beds in the U.S. (Ferguson et al., 2020). If individual reaction to infection risk is weaker or delayed (intuitively meaning that high-risk individuals are not sufficiently willing to avoid infection), the number of complications increase. In this case, government intervention extends the duration of the pandemic, but total deaths are relatively unchanged unless there are technological advances to treat the disease during this time.

The total number of infections and infection complications are substantially lower than the predictions based on simulations in which the individuals are naive because there are big differences in the incentives that different individuals face. For example, Ferguson et al. (2020) predict around 2.2 million deaths if governments take no action. However, under 
the assumptions in the present paper, the individuals that are voluntarily exposed to the virus (and infected) are the low risk types. These individuals are numerous enough in the U.S. to generate "herd immunity" for the entire population once the epidemic wave is over, even for the limit $r_{0}=2.6$ considered by Ferguson et al. (2020). As it is well known in the epidemiology literature, termination of the epidemic does not require all susceptible individuals to be infected. This potentially prevents approximately 100 million infections in the U.S.. Most importantly, none of the high-risk individuals become infected at any time.

The results that I obtain contrast sharply with the combination of mitigation and suppression in Ferguson et al. (2020) and similar models: In these models, governmentimposed confinement temporarily reduces the number of contacts between infectious and susceptible people, while assuming no individual response to infection risk. Once the measures are lifted, the epidemic starts again, unless there are technological advances in treatment during this period. The fixed point in which nobody is infected is unstable if there are enough susceptible individuals left: A perturbation out of the state with zero infected individuals eventually leads to a new epidemic event (Eubank et al., 2020). In addition, the fraction of the population that eventually becomes infected (AR) is typically not very sensitive to $r_{0}$ as long as it is above 1 (Eubank et al., 2020). For example, in the basic model (Ball, 1983),

$$
r_{0}=\ln \left(\frac{1-A R}{A R}\right)
$$

The fundamental difference between the original models and the present one is the composition of the population infected: Infection is heavily skewed towards low risk individuals according to the assumptions that I make, but not in previous models.

Finally, it is possible that some high-risk individuals initially choose to maintain social interaction despite their greater risk of complications. Under the assumption that healthcare capacity is exceeded, the circulation of these high-risk individuals could generate a negative externality on all circulating individuals. Even in this case government intervention can be unnecessary if it is possible to transfer patients from one location to another or if healthcare capacity can be expanded. Indeed, a stylized fact missing from the standard epidemic model 
is that the epidemic peaks at different times in different locations (while these models have a single peak). This implies that the larger the country, the more these models overestimate the national peak in healthcare demand. In addition, the incentives for self-interested high-risk individuals to avoid social contact becomes even stronger if there is a threat of shortage in healthcare. This also contributes to solve the externality problem without government intervention. I develop this robustness analysis in Section 3.3.

In conclusion, the argument that free enterprise results in negative externalities cannot be justified without strong assumptions about individual self-interested reaction to infection risk. In the presence of such assumptions, an important question arises about whose individual preferences government choices represent: It seems challenging to conciliate the assumption that population confinement (in addition to large socio-economic losses) reflect the preferences of the median individual, while self-interested individuals with much higher risk of complications would prefer the risk of infection to self-isolation.

\section{Data and data description}

From the U.S. Census Bureau (2018), I obtain the number of individuals in the U.S. in nine age groups: 0-9, 10-19, 20-29, 30-39, 40-49, 50-59, 60-69, 70-79, and above 80. Column "Until age" in Table 1 shows the upper age limit of these groups, which I index by

$$
g \in\{9,19,29,39,49,59,69,79,89+\}
$$

while columns Pop and Cumulative show the population (in millions) of each age group and the total population with age below and including that group, respectively. The cumulative population shows that the median individual belongs to the age group 39. And the median individual is in group 49 if we only consider every individual above 20.

The remaining columns in Table 1 are conditional probabilities (in percents) and come mostly from Ferguson et al. (2020) and Verity et al. (2020). The conditional probabilities of hospitalization given the presence of symptoms, $P(H \mid S)$, and critical care given hos- 
pitalization, $P(V \mid H)$, are obtained directly from Table 1 in Ferguson et al. (2020). The fatality probability given critical care, $P(D \mid V)$, is calculated to match the infection fatality rate among symptomatic patients (also from Ferguson et al. (2020), Table 1), under the assumption of no mortality in the previous stages.

The next three columns display these probabilities conditioned on infection. The extra information needed in this calculation is the probability of symptoms given infection. Verity et al. (2020) estimate this value to be between 50\% and 60\% (Ferguson et al., 2020), and I consider that $60 \%$ of infections are symptomatic. Hence, the probabilities in the last three columns in Table 1 assume the highest number of complications.

Table 1: Descriptive statistics of Census and epidemiological data. The table displays information corresponding to each age bracket, for which the upper bound is shown in column "Until age". Columns "Pop" and "Cumulative" (in millions) display, respectively, the number of people in each age bracket and the total below and including that age bracket based on the U.S. Census 2018 (American Community Survey). All other columns are conditional probabilities (in percents) in which $H$ is hospitalization, $S$ is symptomatic, $V$ is critical care, $D$ is deceased, and $I$ is infected. Hence, the first three are the probabilities of being (i) hospitalized given the existence of symptoms, (ii) in critical care given hospitalization, and (iii) deceased given critical care. The last three are the probabilities of hospitalization, critical care, and deceased given infection (with 60\% probability of symptoms given infection). The Cumulative column shows that the median individual is in bracket 39 (49 if we ignore the ones below 20).

\begin{tabular}{lrrrrrrrr}
\hline Until age & Pop(mi) & Cumulative & $P(H \mid S)$ & $P(V \mid H)$ & $P(D \mid V)$ & $P(H \mid I)$ & $P(V \mid I)$ & $P(D \mid I)$ \\
\hline 9 & 39.5 & 39.5 & 0.1 & 5.0 & 40.0 & 0.06 & 0.003 & 0.0012 \\
19 & 42.8 & 82.3 & 0.3 & 5.0 & 40.0 & 0.18 & 0.009 & 0.0036 \\
29 & 45.0 & 127.3 & 1.2 & 5.0 & 50.0 & 0.72 & 0.036 & 0.0180 \\
39 & 43.6 & 170.9 & 3.2 & 5.0 & 50.0 & 1.92 & 0.096 & 0.0480 \\
49 & 40.7 & 211.6 & 4.9 & 6.3 & 49.0 & 2.94 & 0.185 & 0.0900 \\
59 & 42.5 & 254.1 & 10.2 & 12.2 & 48.0 & 6.12 & 0.747 & 0.3600 \\
69 & 37.8 & 291.9 & 16.6 & 27.4 & 48.0 & 9.96 & 2.729 & 1.3200 \\
79 & 22.8 & 314.7 & 24.3 & 43.2 & 49.0 & 14.58 & 6.299 & 3.0600 \\
$89+$ & 12.5 & 327.2 & 27.3 & 70.9 & 48.0 & 16.38 & 11.613 & 5.5800 \\
\hline
\end{tabular}

The values in Table 1 are revealing about the average risk that individuals in each age bracket face and the maximum externalities that each group can impose on the others. Under the (extreme) assumption that every individual in a given age bracket is infected, Fig. 1 shows the total number of infected individuals (Population graph), the expected numbers of individuals that require hospitalization (Hospitalized), critical care (Critical), and fatalities (Deceased). 
For example, Fig. 1 reveals that the absolute number of complications (especially critical care and deaths) reported in Ferguson et al. (2020) as a general phenomenon is in fact a fairly delimited issue, that is overwhelmingly dependent on the behavior of a relatively small subset of individuals, in the three smallest age groups $(69,79$, and $89+)$.
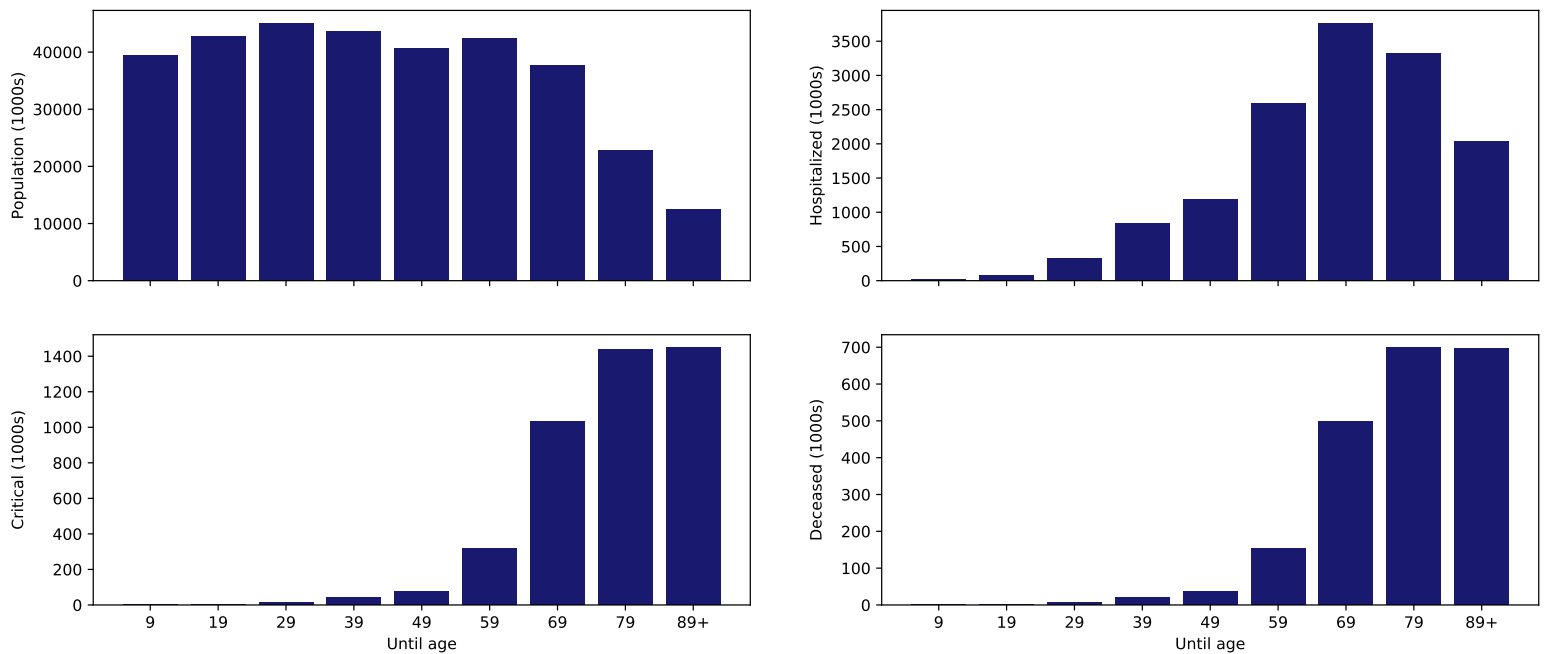

Figure 1: Population and expected number of individuals hospitalized, in critical care, and deceased if everyone is infected, by age group. The population data are from the U.S. Census 2018 (American Community Survey). The remaining values correspond to the population in each group multiplied by the respective probabilities of being hospitalized, in critical care, and deceased given infection (in Table 1).

Fig. 2 further illustrates that the individuals below the age of 49 have a negligible contribution to the use of healthcare services. This suggests that eventual negative externalities created by this group of individuals should also be negligible, as long as the individuals in the remaining groups remain isolated during the epidemic wave phase. For example, the median individual, whose preferences I assume that the government represents, is below 39 years old and belongs to the very low risk group that should demand negligible amounts of healthcare services (as Fig. 1 and Fig. 2 show).

\section{Simulation}

Infected individuals remain infectious for 15 days with no incubation period (infected and infectious can be used interchangeably). A fraction of these individuals stay in hospital 

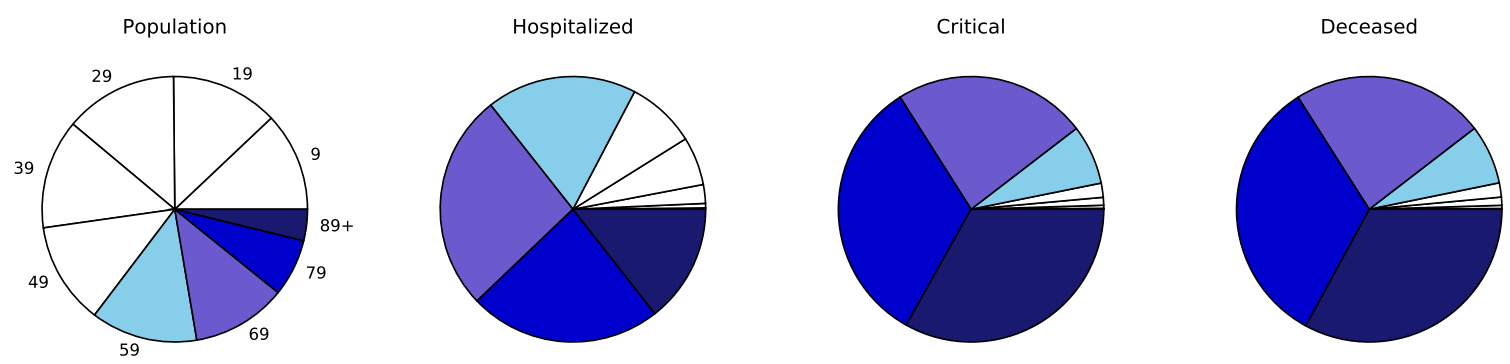

Figure 2: Distribution of population and expected number of individuals hospitalized, in critical care, and deceased if everyone is infected, by age group. The population data are from the U.S. Census 2018 (American Community Survey). The remaining values correspond to the population in each group multiplied by its respective probabilities of being hospitalized, in critical care, and deceased given infection (in Table 1).

for 8 days, and a fraction of those in hospital further stay in critical care for 8 days. These periods are relatively similar to the ones in Ferguson et al. (2020) and they have little impact on the main analysis. For simplicity, fatalities only happen after critical care, while recovery happens after any of the three stages. The basic reproductive number is $r_{0}=2.6$, which is the largest value considered by Ferguson et al. (2020). The simulation starts with 10 newly infected individuals and it lasts for three years.

\subsection{A generalized epidemic framework}

The discrete time deterministic epidemic model that I use is an extension of the compartmental model of Kermack and McKendrick (1927). The model incorporates both self-interested and heterogeneous responses of the individuals to infection risk and also the effects of government-imposed confinement.

The dynamics is easier to understand if we define the number of newly infected individuals each period,

$$
I_{t}^{\prime} \equiv \kappa_{t} I_{t} \frac{S_{t}}{N_{f, t}},
$$

where $\kappa_{t}$ is the number of individuals that each infectious individual could infect per day (intuitively interpreted as "encounters"), $I_{t}$ is the total number of infectious individuals, and the last term is the fraction of susceptible individuals, $S_{t}$, among all the individuals 
that are circulating, $N_{f, t}$, This ratio is related to the proportion of susceptible individuals among the potentially infected (the ones that "encounter" infectious individuals). ${ }^{1}$ A novel feature of the model is that the sum of the circulating population, $N_{f, t}$, and the restricted population, $N_{r, t}$, is the total (constant) population,

$$
N=N_{f, t}+N_{r, t}, \quad \forall t
$$

where individuals choose to belong to the restricted or circulating populations, depending on their individual preferences with respect to infection risk. In addition,

$$
\kappa_{t}=\frac{r_{0, t}}{\ell_{I}}
$$

where $r_{0, t}$ is the reproductive number (which can vary over time, depending on governmentimposed confinement) and $\ell_{I}=15$ is the number of days that each infected individual remains infectious.

After 15 days, part of these individuals recovers (moves to the Recovered compartment, $R_{t}$ ) and the other part moves to the Hospitalized compartment, $H_{t}$, for 8 more days. Regardless of the compartment, the individual stops infecting others after these 15 days. After a total of $\ell_{H}=23$ days from infection, the individuals in the hospital compartment leave either to the Recovered or to the Critical care compartment, $V_{t}$, for 8 extra days. Finally, after a total of $\ell_{V}=31$ days from infection, the individuals in the critical compartment leave either to the Recovered compartment or the Deceased compartment, $D_{t}$.

The fractions of newly infected individuals at a given time, $I_{t}^{\prime}$, that arrive at the Hospital, Critical, and Deceased compartments, respectively, $\ell_{I}=15, \ell_{H}=23$, and $\ell_{V}=31$ days later are equal to the probabilities given infection of hospitalization, $P_{t}(H \mid I)$, critical care, $P_{t}(V \mid I)$, and death, $P_{t}(D \mid I)$ in Table 1 . The remaining individuals move to the Recovered compartment at these periods. In the baseline formulation, in which the composition of the circulating population changes because the individuals respond to the risk of infection

\footnotetext{
${ }^{1}$ However, it ignores that different infected individuals can encounter the same group of susceptible individuals.
} 
with self-isolation, these probabilities are time-varying. They are the weighted averages of the values in Table 1, with weights given by the number of individuals in each age bracket circulating at time $t$. For example, the aggregate probability of hospitalization given infection at time $t$ (ignoring the time subscript in all variables to save on notation) is given by

$$
P(H \mid I)=\frac{\sum_{g=9}^{89+} n_{f, g} P_{g}(H \mid I)}{N_{f}},
$$

where the sum is taken across the nine age groups, $g$ in Eq. (2), $n_{f, g}$ is the number of circulating individuals from each age group $g, N_{f}$ is the total number of circulating individuals, and $P_{g}(H \mid I)$ is the probability of hospitalization given infection of individuals of age group $g$ (listed in Table 1).

The evolution of the number of individuals in each compartment is formally given by

$$
\begin{aligned}
S_{t+1} & =S_{t}-I_{t}^{\prime}+\left(N_{f, t}-N_{f, t-1}\right), \\
I_{t+1} & =I_{t}+I_{t}^{\prime}-I_{t-\ell_{I}}^{\prime}, \\
H_{t+1} & =H_{t}+I_{t-\ell_{I}}^{\prime} P_{t-\ell_{I}}(H \mid I)-I_{t-\ell_{H}}^{\prime} P_{t-\ell_{H}}(H \mid I), \\
V_{t+1} & =V_{t}+I_{t-\ell_{H}}^{\prime} P_{t-\ell_{H}}(V \mid I)-I_{t-\ell_{V}}^{\prime} P_{t-\ell_{V}}(V \mid I), \\
D_{t+1} & =D_{t}+I_{t-\ell_{V}}^{\prime} P_{t-\ell_{V}}(D \mid I), \\
R_{t+1} & =R_{t}+I_{t-\ell_{I}}^{\prime}\left(1-P_{t-\ell_{I}}(H \mid I)\right) \\
& +I_{t-\ell_{H}}^{\prime}\left(P_{t-\ell_{H}}(H \mid I)-P_{t-\ell_{H}}(V \mid I)\right)+I_{t-\ell_{V}}^{\prime}\left(P_{t-\ell_{V}}(V \mid I)-P_{t-\ell_{V}}(D \mid I)\right),
\end{aligned}
$$

where the number of susceptible individuals in Eq. (7) include the change in the number of individuals circulating from one period to the next (in brackets). There is no re-infection, as in Kermack and McKendrick (1927).

\subsection{Four main scenarios}

This section investigates four stylized scenarios. In two of them, there is no individual reaction to infection risk. There is government-imposed confinement in the "Confined" 
scenario, but not on the "Naive" scenario. In the other two, self-interested individuals react to infection risk: There is government-imposed confinement in the "Confined-res" scenario, but individuals are free on the "Response" scenario.

Response: In the baseline simulation (Response), every individual remains free and the government does nothing. By assumption, from the start of the simulation, all individuals above the age of 49 find optimal to self-isolate from the rest of the population in response to the threat of infection. This assumption is based on the fact that these individuals face substantially higher risk of complications than the one faced by the median individual (Table 1). They remain isolated until the number of critical cases is below 1. At this point, everyone circulates. The (time-varying) fractions of individuals that transition to each compartment in the simulation are given by the weighted average of the probabilities listed in Table 1, based on the circulating population: The weights are the number of circulating individuals in each age bracket, as illustrated by Eq. (6).

Naive: In the Naive simulation, individuals are still free, but they do not adjust their behavior to avoid infection. This simulation is similar to the "unmitigated" scenario imagined by Ferguson et al. (2020).

Confined: In the Confined simulation, the government forces the entire population into confinement for two years and reduce the reproductive number, $r_{0}$, during this period. There are two regimes during this period: If the daily number of new arrivals in critical care rises above 125, stronger confinement lowers social contact and the reproductive number by $70 \%$, to $0.3 r_{0}$. This regime is in place about $58 \%$ of the time. If the number of new arrivals in critical care falls below 25 (while decreasing), confinement lowers social contact and the reproductive number by $50 \%$, to $0.5 r_{0}$. This formulation is similar to the "ON/OFF" policy proposed by Ferguson et al. (2020), scaled for the U.S. population and critical care capacity. After two years, I assume that the government frees the population, which is a step that is not addressed by Ferguson et al. (2020). No new treatments are available at 
this time, and individuals do not try to avoid infection. Hence, the simulation reverts to the "Naive" scenario.

Confined-res: The Confined-res simulation is a mix between the Confined and Response simulations: The government forces the population into confinement for two years, and the individuals above 49 still choose to self-isolate until the number of critical cases is below one. After two years, this simulation also reverts to the "Naive" scenario for comparison with the Confined case.

\subsubsection{Critical and deceased individuals}

Fig. 3 displays the results of these four scenarios during the first two years (on the lefthand side graphs) and during the full three years (on the right-hand side graphs). I only include the Naive simulation on the full period graphs (due to magnitude differences). The simulation starts in January 12020 with 10 infected. The Response simulation is in yellow, Confined is in blue, Confined-res is in pink, and Naive is in purple. The figure displays the number of individuals in critical care (bottom graphs) and deceased (top graphs).

If the individuals are free, there is only one epidemic event. In the Naive simulation, there is a very large surge in maximum critical care and total deceased individuals, to approximately 1.2 and 2 million, respectively. The values (which I only report in the 3-year graphs) are two orders of magnitude higher than the ones in the Response simulation: Crit-

ical cases and deaths surge around May to approximately 35 and 60 thousand, respectively, and the epidemic terminates around July.

In the presence of government-mandated confinement, there are multiple epidemic events. In the Confined-res simulation, the total number of critical cases and deaths surge to around 15 and 20 thousand, respectively, and remain close to these values while the population is confined. The individuals above 49 years respond to the risk of infection by further self-isolating during most of this period (as I show later). When the government 

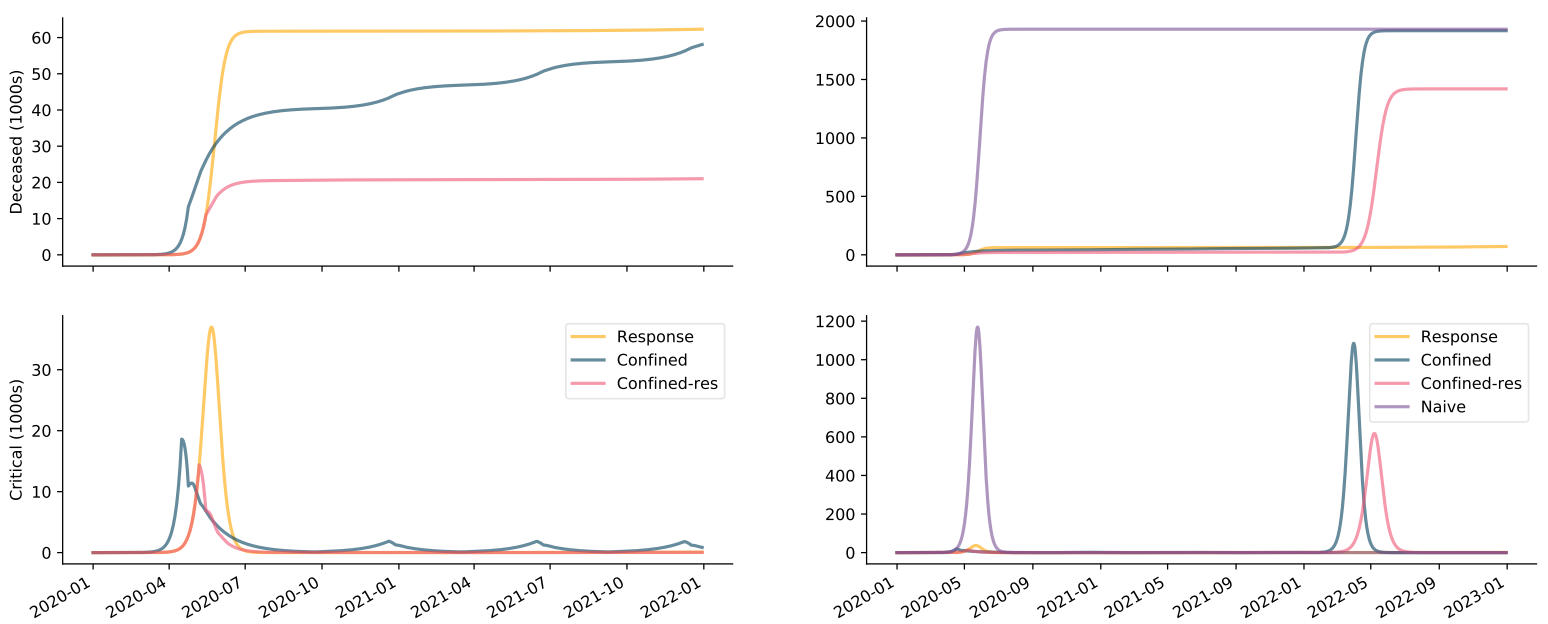

Figure 3: Critical and Deceased individuals with or without government-imposed confinement and individual reaction to infection risk. Early period on the left, full simulation on the right (population is free after 2 years). The compartments in the simulation are: Susceptible, infected, hospitalized, critical (shown in the bottom graphs), recovered, and deceased (in the top graphs). The left-hand side graphs show the first 2 simulated years, with possible governmentimposed confinement. The graphs on the right include the period after the government frees the population (if everyone circulates). The simulation starts in January 12020 with 10 infected, $r_{0}=2.6,15$ infectious days, 8 days in hospital, and 8 days in critical care. There are no reinfections and death can only occur after all stages. The fractions of individuals transitioning to the next compartments are given by aggregating the probabilities in Table 1 (by age group). The remaining assumptions are: In yellow (Response) only the individuals above 49 years react to infection risk and isolate from everyone until the number of critical patients is below 1 . In blue (Confined), the government confines the entire population, but no individual reacts to infection risk. During confinement, the government restricts social contact of all individuals by $70 \%$, $r_{0, \text { on }}=0.3 r_{0}$, if the daily number of critical patients is above 125 or if it is decreasing but above 25 . In the remaining of these two years, around $42 \%$ of the time, the government restricts social contact by $50 \%, r_{0, o f f}=0.5 r_{0}$. In pink (Confined-res), the government confines the population and the individuals above 49 years react to infection risk and isolate from everyone until the number of critical patients is below 1 . In purple (Naive), there is no change in individual behavior nor government restrictions.

frees the population, there is a large second epidemic, which leaves total deaths at almost 1.5 million, for example.

In the Confinement simulation, critical cases surge to less than 20 thousand, followed by three smaller epidemic events until 2022, each one triggers a switch from one type of confinement to another. The total number of deaths increases slowly during two years and almost reaches the same 60 thousand obtained in the Response simulation. After two years, there is a large epidemic with virtually the same size of the Naive simulation. The total number of deaths is virtually the same in the Naive and Confinement simulations. 


\subsubsection{Susceptible and infected individuals}

Fig. 4 is equivalent to Fig. 3 but shows the number of infected and susceptible (circulating) individuals. This further explains the differences among the four scenarios. For example, Fig. 4 shows that government-imposed confinement strongly prevents the number of infected individuals to increase in the Confinement simulation: The number of susceptible individuals remains almost the same while the population is confined. As a consequence the number of immune individuals remains negligible. This is why total deaths and the maximum number of critical individuals are virtually the same in the Naive and Confinement simulations after the government frees the population in Fig. 3.

If the population is free, in the Response simulation, the number of infected individuals peaks at approximately 100 million around May, and the dynamics almost exhausts the number of susceptible individuals in the circulating population. Around September, the number of critical cases is below one, and the high-risk individuals start to circulate. This increases the total of susceptible individuals. Nevertheless, there is no further epidemic because the fraction of susceptible individuals in the total population is not large enough. The population achieves "herd immunity" without infecting any of the individuals with high risk of complications.

However, if the government forces the population into confinement for two years, in the Confinement-res simulation, the number of infected individuals never rises above 30 million and there is only a small decrease in the number of susceptibles. When the highrisk individuals start to circulate again, around July 2021, almost the entire circulating population is susceptible. Hence, a large epidemic takes place again when the government frees the population. Naturally, it is possible to assume that high risk individuals decide to self-isolate again in the third year. In this case (not shown), this second epidemic, in the third year, is simply a smaller version of the single epidemic in the Response simulation.

Finally, approximately 50 million individuals reach the end of the epidemic without being infected in the Confinement-res simulation, which is more than in the Naive and 

million) avoid being infected in the Response simulation.
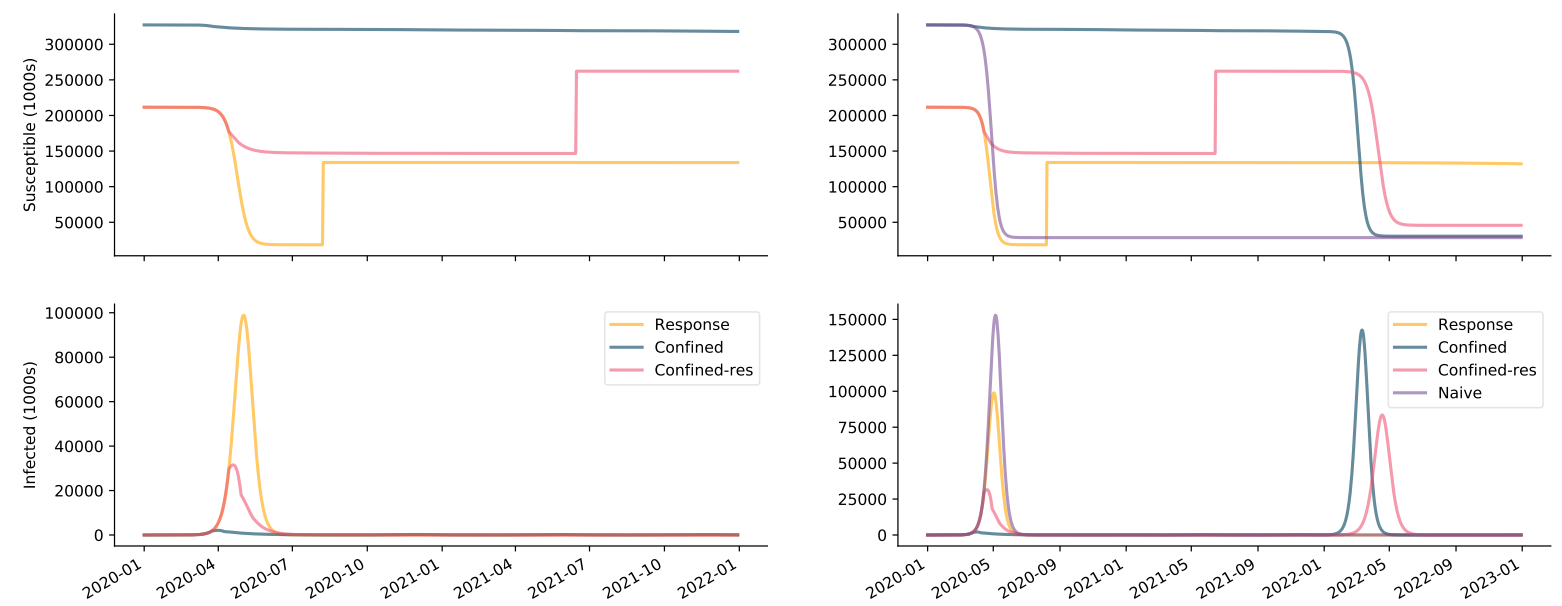

Figure 4: Susceptible and Infected individuals with or without government-imposed confinement and individual reaction to infection risk. Early period on the left, full simulation on the right (population is free after 2 years). The compartments in the simulation are: Susceptible (in the top graphs), infected (in the bottom graphs), hospitalized, critical, recovered, and deceased. The left-hand side graphs show the first 2 simulated years, with possible governmentimposed confinement. The graphs on the right include the period after the government frees the population (if everyone circulates). The simulation starts in January 12020 with 10 infected, $r_{0}=2.6,15$ infectious days, 8 days in hospital, and 8 days in critical care. There are no reinfections and death can only occur after all stages. The fractions of individuals transitioning to the next compartments are given by aggregating the probabilities in Table 1 (by age group). The remaining assumptions are: In yellow (Response) only the individuals above 49 years react to infection risk and isolate from everyone until the number of critical patients is below 1 . In blue (Confined), the government confines the entire population, but no individual reacts to infection risk. During confinement, the government restricts social contact of all individuals by $70 \%$, $r_{0, \text { on }}=0.3 r_{0}$, if the daily number of critical patients is above 125 or if it is decreasing but above 25 . In the remaining of these two years, around $42 \%$ of the time, the government restricts social contact by $50 \%, r_{0, \text { off }}=0.5 r_{0}$. In pink (Confined-res), the government confines the population and the individuals above 49 years react to infection risk and isolate from everyone until the number of critical patients is below 1 . In purple (Naive), there is no change in individual behavior nor government restrictions.

\subsection{Variations in the Response scenario}

This section documents four variations of the Response scenario described in Section 3.2. Fig. 5 displays the number of deceased (on the left) and critical individuals (on the right) during the first seven simulated months for each of these variations. The epidemic is over after this period in all of them. 
The Response simulation (in green) is exactly as described in Section 3.2: All (and only) individuals above 49 years react to the threat of infection by self-isolating from the start of the simulation until the number of critical patients is below 1. Individuals are free in all simulations. The other four scenarios are as follows:

- $49^{+}$(late): Displayed in pink in Fig. 5, the simulation starts with all individuals circulating, until the first patient arrives in critical care. From this point, the simulation is equivalent to the Response simulation: Individuals above 49 self-isolate until the number of critical patients is below 1 .

- $59^{+}$: In orange, all (and only) individuals above 59 years react to infection risk and self-isolate since the beginning of the simulation until the number of critical patients is below 1 .

- $49^{+}(90 \%)$ : In yellow, $90 \%$ of the individuals above 49 years (and only those) react to infection risk and self-isolate since the beginning of the simulation until the number of critical patients is below 1 .

- $49^{+}(70 \%)$ : In purple, $70 \%$ of the individuals above 49 years (and only those) react to infection risk and self-isolate since the beginning of the simulation until the number of critical patients is below 1 .

These variations of the Response scenario illustrate a few points. For example, the $49^{+}$ (late) simulation shows that if high-risk individuals only self-isolate after observing the first critical patient, the number of total deaths nearly doubles and the peak in critical cases nearly triples compared to the Response scenario (both to around 100 thousand).

For the $49^{+}(70 \%)$ simulation, deceased and peak in critical individuals are above $600 / 350$ thousand, respectively. This is approximately 10 times the values in the Response simulation. However, the social restrictions on the individuals modeled in the $49^{+}(70 \%)$ simulation are similar to the confinement plan that reduces social interaction by $70 \%$ imposed by the government on the entire population. Hence, I expect the values obtained 
in this simulation to overestimate the number of complications, as long as the government does not confine the entire population.

In addition, the numbers of deceased and critical individuals grow relatively fast as the proportion of isolated individuals above 49 lowers. If even only $10 \%$ of these individuals choose to continue social interaction, in the $49^{+}(90 \%)$ simulation, the peak number of critical and total deceased individuals are about 150/280 thousand, which is substantially larger than the 35/60 thousand if this fraction is zero, in the Response simulation. Finally, if all (and only) the individuals above 59 self-isolate, these numbers are a bit lower, but still substantially higher than the values in the Response simulation.
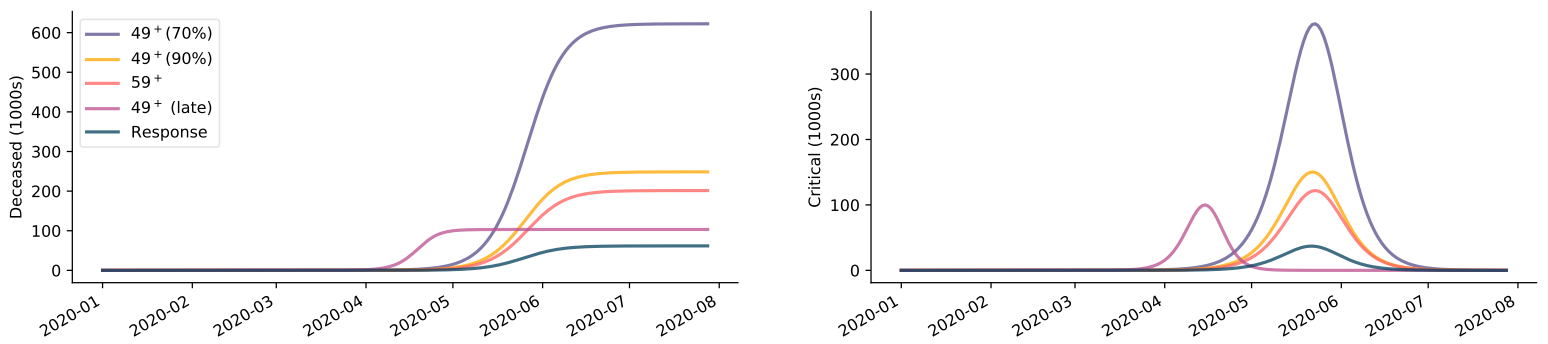

Figure 5: Outbreak simulations in a free population, based on different individual reactions to infection risk (first 7 months). The compartments in the simulation are: Susceptible, infected, hospitalized, critical (right-hand side graph), recovered, and deceased (left-hand side graph). The simulations start in January 12020 with 10 infected, $r_{0}=2.6,15$ infectious days, 8 days in hospital, and 8 days in critical care. There are no reinfections and death can only occur after all compartments. The fractions of individuals transitioning to the next compartments are given by aggregating the probabilities in Table 1 (by age group), depending on the composition of the exposed population each period. The particular assumptions are: In green (Baseline $49^{+}$), all (and only) individuals above 49 years react to infection risk and isolate from everyone since the beginning of the simulation until the number of critical patients is below 1 . In pink ( $49^{+}$(late)), all individuals remain exposed until the first patient arrives in critical care. From this point, the simulation is equivalent to the baseline: Individuals above 49 isolate from everyone until the number of critical patients is below 1 . In orange $\left(59^{+}\right)$, all (and only) individuals above 59 years react to infection risk and isolate from everyone since the beginning of the simulation until the number of critical patients is below 1 . In yellow $\left(49^{+}(90 \%)\right), 90 \%$ of the individuals above 49 years (and only those) react to infection risk and isolate from everyone since the beginning of the simulation until the number of critical patients is below 1 . In purple $\left(49^{+}(70 \%)\right), 70 \%$ of the individuals above 49 years (and only those) react to infection risk and isolate from everyone since the beginning of the simulation until the number of critical patients is below 1 . This last formulation can be broadly compared to government-imposed confinement that reduces aggregate social interaction by $70 \%$, but only applied to the population above 49 years in this case. 


\section{Summary}

In this paper we learn that the argument that government-imposed confinement can be justified based on hypothetical negative externalities that low-risk individuals generate for high-risk individuals requires very strong assumptions about the responses that these high-risk individuals have to the threat of infection. Paradoxically, the argument requires that self-interested high-risk individuals do not see infection as sufficiently undesirable.

Otherwise, we learn that the circulation of low risk individuals in fact generate strong positive externalities in the form of "herd immunity". And we learn that governmentimposed confinement prohibits low risk individuals from generating this positive effect.

Hence, government-imposed confinement during the Coronavirus outbreak in the U.S. is an example of government failure under the assumption that high-risk individuals perceive infection as sufficiently undesirable, even if we ignore the socio-economic costs associated with the destruction of businesses, jobs, and violations of individual rights. 


\section{References}

Ball, Frank, 1983, A threshold theorem for the reed-frost chain-binomial epidemic, Journal of Applied Probability 20, 153-157.

Dawson, John W., and John J. Seater, 2013, Federal regulation and aggregate economic growth, Journal of Economic Growth 18, 137-177.

de Oliveira Souza, Thiago, 2020, Consumption, government failure, and asset prices, Unpublished working paper. University of Southern Denmark.

Eubank, S., I. Eckstrand, B. Lewis, S. Venkatramanan, M. Marathe, and C. L. Barrett, 2020, Commentary on Ferguson, et al., "Impact of non-pharmaceutical interventions (NPIs) to reduce COVID-19 mortality and healthcare demand", Bulletin of Mathematical Biology 82.

Ferguson, Neil, Daniel Laydon, Gemma Nedjati Gilani, Natsuko Imai, Kylie Ainslie, Marc Baguelin, Sangeeta Bhatia, Adhiratha Boonyasiri, ZULMA Cucunuba Perez, Gina CuomoDannenburg, et al., 2020, Impact of non-pharmaceutical interventions (NPIs) to reduce COVID19 mortality and healthcare demand, Techical report, doi: 10.25561/77482.

Ferguson, Neil M, Derek AT Cummings, Christophe Fraser, James C Cajka, Philip C Cooley, and Donald S Burke, 2006, Strategies for mitigating an influenza pandemic, Nature 442, $448-452$.

Halloran, M Elizabeth, Neil M Ferguson, Stephen Eubank, Ira M Longini, Derek AT Cummings, Bryan Lewis, Shufu Xu, Christophe Fraser, Anil Vullikanti, Timothy C Germann, et al., 2008, Modeling targeted layered containment of an influenza pandemic in the united states, Proceedings of the National Academy of Sciences 105, 4639-4644. 
Kermack, W. O., and A. G. McKendrick, 1927, A contribution to the mathematical theory of epidemics, Proceedings of the Royal Society of London. Series A, Containing Papers of a Mathematical and Physical Character 115, 700-721.

McKean, Roland N., 1965, The unseen hand in government, American Economic Review 55, 496-506.

U.S. Census Bureau, 2018, American Community Survey: 1-year estimates subject tables, Table ID: S0101.

Verity, Robert, Lucy C Okell, Ilaria Dorigatti, Peter Winskill, Charles Whittaker, Natsuko Imai, Gina Cuomo-Dannenburg, Thompson, et al., 2020, Estimates of the severity of covid-19 disease, medRxiv doi: 10.1101/2020.03.09.20033357 .

Wolf, Charles, 1979, A theory of nonmarket failure: Framework for implementation analysis, Journal of Law \& Economics 22, 107-139. 